\title{
Caspase-3 may be employed as an early predictor for fracture-induced osteonecrosis of the femoral head in a canine model
}

\author{
YOU-SHUI GAO, SHANG-CHUN GUO, HAO DING and CHANG-QING ZHANG \\ Department of Orthopaedic Surgery, Shanghai Sixth People's Hospital, \\ Shanghai Jiao Tong University, Shanghai 200233, P.R. China
}

Received February 24, 2012; Accepted June 15, 2012

DOI: $10.3892 / \mathrm{mmr} .2012 .958$

\begin{abstract}
The aim of the current study was to investigate the local expression of caspase-3 following femoral neck fractures in a canine model and to investigate its effect on the occurrence of fracture-induced osteonecrosis of the femoral head (ONFH). Eight dogs had surgically-induced femoral neck fractures on the left side which remained untreated. Radiological and histological examinations were employed to detect morphological changes of the femoral head. Immunohistochemical staining of caspase-3 was used to evaluate cell apoptosis, which may play an important role in ONFH. The results were compared to the normal side for statistical analysis. As a result, all eight dogs had ONFH, with non-union in five and malunion in three on radiological examination. Histologically, the untreated femoral heads developed osteonecrosis with an accumulation of bone marrow cell debris, empty lacunae and/or ghost nuclei in the lacunae, and an increase in the number of fat cells. Immunohistochemical staining of caspase-3 indicated that it was upregulated in fracture-induced ONFH two weeks postoperatively, which showed a statistical difference when compared to the normal side. In conclusion, the local expression of caspase-3 was upregulated in fracture-induced ONFH, suggesting that cell apoptosis is crucial in traumatic ONFH. Caspase- 3 may therefore be employed as an effective and early predictor for fracture-induced ONFH.
\end{abstract}

\section{Introduction}

Fracture-induced osteonecrosis of the femoral head (ONFH) is a severe complication following femoral neck fractures (1). Reportedly, fracture-induced ONFH occurs in approximately

Correspondence to: Professor Chang-Qing Zhang, Department of Orthopaedics, Shanghai Sixth People's Hospital, Shanghai Jiao Tong University, Shanghai 200233, P.R. China

E-mail: changqing_1914@yahoo.cn

Key words: caspase-3, osteonecrosis of the femoral head, femoral neck fractures, canine model, immunohistochemistry
$17.5 \%$ of all surgically-treated patients with femoral neck fractures (1-3). As the pathogenesis of ONFH has not been elucidated clearly, there are various treatment protocols for the disease with respective indications, including core decompression, structural and non-structural bone grafting, vascularized and non-vascularized bone implantation, porous metal grafting and osteotomy, as well as artificial joint replacement (4-6). It remains controversial as to which method is optimal, as none of these surgical techniques are perfect. However, it has been proven that an early-stage ONFH achieves a better prognosis irrespective of which type of head-preserving surgery is employed (7). Therefore, it appears critical to confirm fracture-induced ONFH at its early stage, in an accurate and repeatable manner.

Previously, several authors attempted radiological and surgical methods to predict fracture-induced ONFH (8-12); however, the shortcomings of these methods have restricted their clinical application. Traditionally, histological examination has been considered as the gold standard for clinical diagnosis, including cellular and extracellular pathogenesis in particular. Additionally, immunohistochemical methods have been widely employed to detect cell apoptosis in modern pathology. Apoptosis may play an important role in the pathogenesis of ONFH induced by steroids and alcohol (13), and may be a significant cause of bone cell death in ONFH, which is associated with apoptosis (14). Thus, whether apoptosis also plays a similar role in fracture-induced ONFH remains to be determined.

In the current study, a canine model of femoral neck fractures was made and evaluated. Within different post-fracture intervals, the femoral head was collected for histological examination and caspase-3 staining, which controls both cytoplasmic and nuclear events associated with Fas-mediated apoptosis in vivo (15). The present study was approved by the ethics committee of Shanghai Sixth People's Hospital.

\section{Materials and methods}

Animals. Beagles from the Laboratory Animal Center of the Shanghai Sixth People's Hospital, China, were used as the canine model and had an average age of 2.5 years and body weight of $14.5 \mathrm{~kg}$ (range, 13-17 kg). The animals had no 
history of disease and were housed separately. Before the day of surgery, the dogs were fasted, and penicillin sodium was injected intramuscularly. A total of eight dogs were used in the present study.

Surgical methods and sample acquisition. Under aseptic conditions, a 6 -cm-long L-shape skin incision was made centered to the left of the great trochanter to expose the hip joint. The tensor fascia lata was split along the length of its bundles, and the gluteus medius muscle was detached partially from the great trochanter. The hip joint capsule was opened, and electrocoagulation of soft tissue attachments at the base of the femoral neck was performed circumferentially to interrupt the extraosseous blood supply to the femoral head. However, the medial and lateral circumflex arteries were not revealed, and the ligamentum teres was kept intact. A low-speed drill was employed to fracture the femoral neck at the narrow base. The femoral neck fractures were left untreated to induce avascular osteonecrosis. The maneuvers were directly visible for assurance of anatomical reduction and reliable stabilization. Surgical procedures were completed by one team of surgeons. Postoperatively, the animals were housed separately and injected with penicillin sodium for prophylactic control of infection. The right femoral head was set for the control $(\mathrm{N}=8)$.

Radiological and histological examinations. The animals underwent radiological examination two weeks postoperatively and then monthly on three occasions. After each radiological examination, two animals were euthanized, and the bilateral femoral heads were collected for histological examination. For the microscopic examination, tissue samples were obtained from the zone of weight-bearing and the center of the femoral head. The samples were fixed with $10 \%$ formalin for one week and decalcified with $5 \mu \mathrm{M}$ EDTA solution for four weeks. The specimens were embedded in paraffin, cut into $4-\mu \mathrm{m}$ sections, and stained with hematoxylin and eosin (H\&E).

Immunohistochemistry of caspase-3 expression. For immunohistochemistry, each section was de-waxed, irradiated at $750 \mathrm{~W}$ in a microwave oven with $3 \%$ hydrogen peroxide in $0.01 \mathrm{M}$ sodium citrate buffer ( $\mathrm{pH}$ 6.0) for $5 \mathrm{~min}$, and immunostained with a monoclonal anti-caspase-3 antibody (Hope, Zhenjiang, Jiangsu, China) to detect osteogenesis and osteocyte apoptosis in the femoral head.

Statistical analysis. The numerical data were presented as the means \pm standard deviation (SD). Fisher's exact test was employed to compare the percentage of caspase-3-positive cells of the necrotic femoral head to the reference value using SPSS 17.0 software (Chicago, IL, USA). P $<0.05$ was considered to indicate a statistically significant difference.

\section{Results}

General and radiological examination. None of the animals experienced postoperative complications of infection. Radiologically, five dogs showed non-union and three dogs had malunion of the femoral head. All eight animals had fracture-induced ONFH in their left femoral heads based on last radiological examination.
$\mathbf{A}$

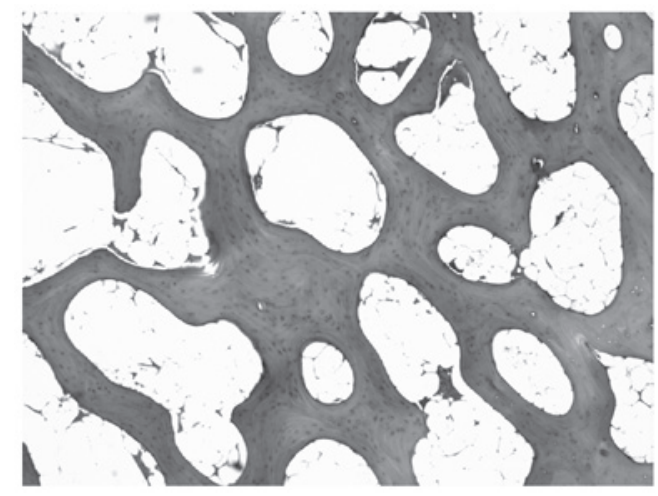

B

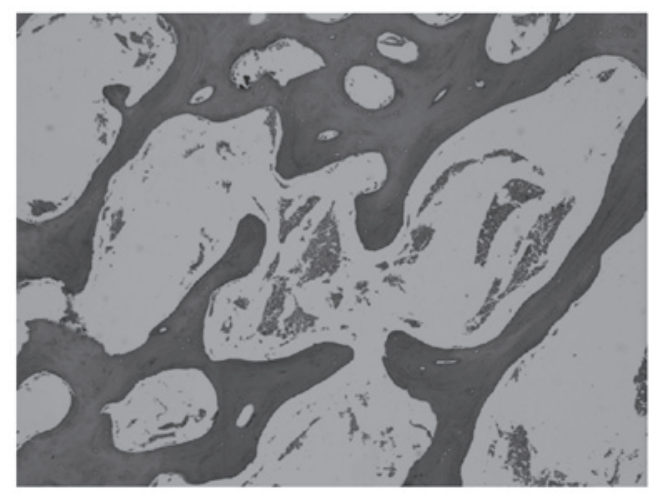

C

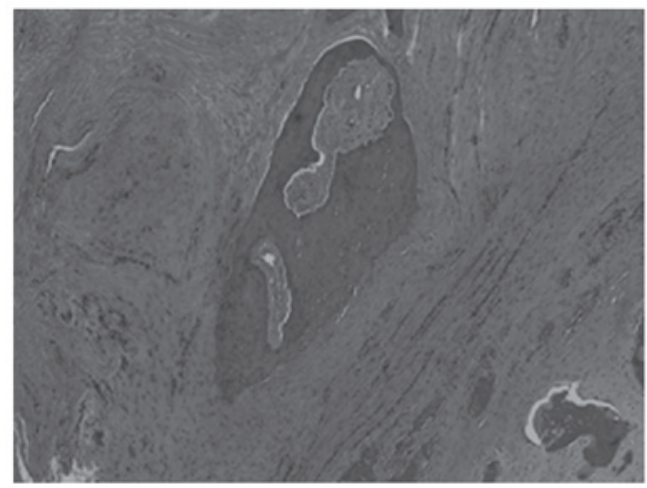

Figure 1. H\&E staining of fracture-induced ONFH. (A) The trabeculae were discrete, with an increase of fat cells in the trabecular space. (B) Empty lacunae and ghost cells were located diffusely in the discrete trabeculae. (C) The bone island was surrounded by fibrous tissue, which was probably stimulated for pathological reparation (x50). H\&E, hematoxylin and eosin; ONFH, osteonecrosis of the femoral head.

Histological examination. Morphologically, the surface of the cartilage lost continuity for untreated femoral neck fractures. Results of the H\&E staining indicated that the untreated head developed osteonecrosis with characteristics of an accumulation of bone marrow cell debris, empty lacunae and/or ghost nuclei in the lacunae, and an increase in fat cells of the bone marrow. Proliferation of fibrous tissue was triggered for pathological reparation as well (Fig. 1A-C).

For the immunohistochemical staining of caspase-3, 10 fields of positive and total cells were randomly counted by two authors, and the percentage was used for statistical analysis. There were eight normal femoral heads, which were set as a reference value. There were $8.5 \pm 2.2 \%$ positive cells in the normal head; however, there were 41, 45.5, 32.5 and $28.5 \%$ positive cells in the osteonecrotic head two weeks, 1.5, 2.5 and 3.5 months following femoral neck fractures, respectively. 
A

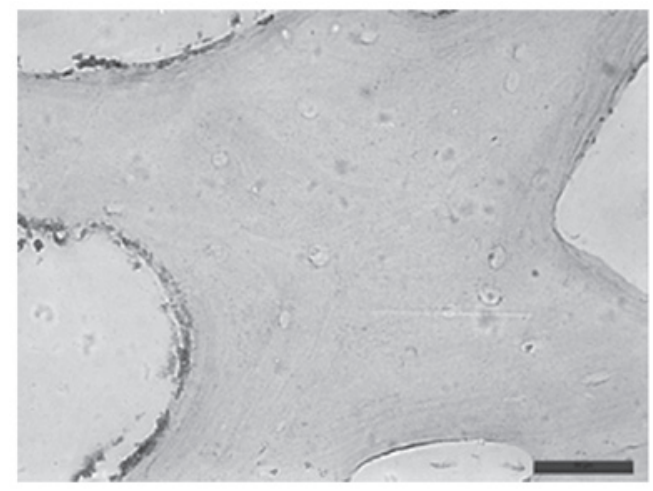

B

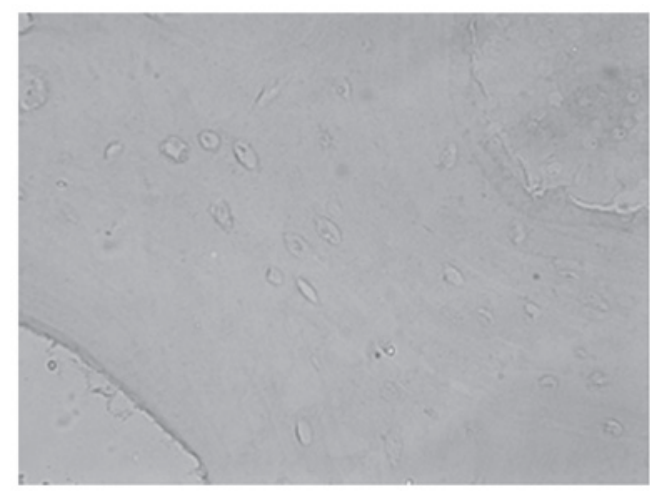

C

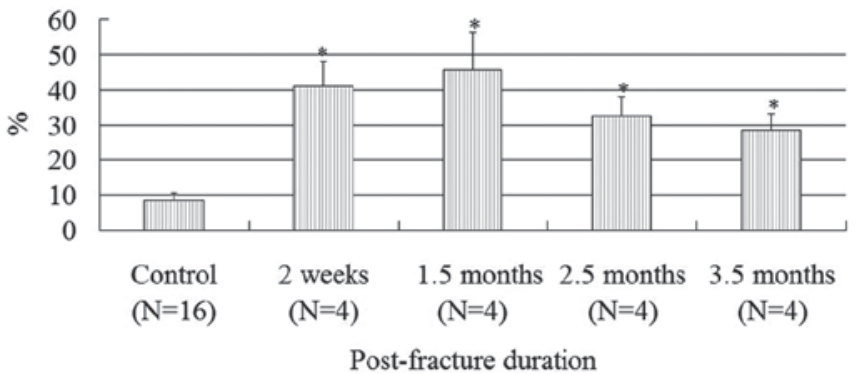

Figure 2. Immunohistochemical staining of caspase-3. (A) There were fewer caspase-3-positive cells in normal femoral head. (B) However, the percentage of caspase-3-positive cells was higher in fracture-induced femoral head two weeks after fracture. (C) For statistical analysis, there were more caspase-3positive cells in osteonecrotic femoral head in comparison to the norma femoral head 2 weeks, 1.5, 2.5 and 3.5 months after fracture $(\mathrm{x} 400)\left({ }^{*} \mathrm{P}<0.05\right)$.

It was found that there were more caspase-3-positive cells in untreated and osteonecrotic femoral head as early as two weeks to 3.5 months following femoral neck fractures, which showed a statistical difference in comparison to the reference value (Fig. 2A-C).

\section{Discussion}

Fracture-induced ONFH is a complication that occurs following femoral neck fractures, due to its unsatisfactory prognosis with current treatment protocols. If the femoral head were to experience biomechanical collapse, the results would be poor, and artificial joint replacement would become inevitable to restore a functional hip. Based on up-to-date clinical reports, pre-collapse ONFH is therefore likely to achieve much better results in comparison with collapsed osteonecrosis when the head is to be preserved (16). Therefore, it is of great significance to make an early diagnosis of ONFH, to facilitate early treatment accordingly. As indicated previously, although there have been various methods to predict fracture-induced $\mathrm{ONFH}$, none of these techniques have been applied in the clinic and further reports regarding such methods are non-existent. In their study, Cho et al found that intraoperative bleeding from the cannulated screws used for the fixation of intracapsular femoral neck fractures could be employed as a predictive method for subsequent ONFH (10). However, evidently, the method may be affected by extrascrew bleeding, and postoperative vascular restoration was neglected. Moreover, for certain intracapsular neck fractures, the optimal implant may not be cannulated screws, which has restricted its broad application (17). Watanabe et al measured the intramedullary oxygen tension of the proximal femur and evaluated its usefulness in monitoring for the prediction of fracture-induced ONFH (9). Although the method was simple and less invasive, it may be affected by various subjective factors, and consecutive monitoring was not be realized. Non-invasive methods, including magnetic resonance imaging (MRI), bone scintigraphy and positron emission tomography (PET), have been advocated for the prediction of ONFH induced by various etiologies. However, clinical application of these methods is limited due to a lack of instruments, the nonquantitative results, as well as the use of radioactive materials. Risk factors for fracture-induced ONFH have been indicated, including primary fracture displacement, the quality of reduction and internal fixation, and operative methods; however, the intrinsic relationship of these factors to the occurrence of ONFH is unclear (18-20).

Although the pathogenesis of ONFH is poorly understood, it has been found that cell apoptosis plays an important role in the initiation and progression of ONFH induced by alcohol and steroids (13). Apoptosis, also known as programmed cell death, is triggered by cellular stress, such as serum deprivation and hypoxia resulting from femoral neck fractures. Sen et al used a core biopsy from the supra-lateral femoral head of patients with unreduced hip dislocation or fracture dislocation and found dead osseous fragments and necrotic osteocytes. Those authors concluded that core biopsy biology as well as marrow-aspirate volume and morphology were capable of predicting the development of subsequent ONFH following trauma (11). During the development of ischemic ONFH, oxygen-regulated protein 150 and haemoxygenase 1 may play an important role in the mechanism of cell apoptosis (21). Similarly, apoptosis of osteocytes was abundantly found in ONFH induced by corticosteroid and hydrogen peroxide (22-25). Caspase-3 controls both cytoplasmic and nuclear events associated with Fas-mediated apoptosis in vivo (15), and previously, the activation of caspase- 3 has been indicated in the initiation of ONFH (20). Histological methods, strengthened by modern immunological means, are traditionally considered as the gold standard for an early diagnosis of this complex disease.

As indicated by results of the present study, caspase- 3 is activated as early as two weeks following femoral neck fractures, and is closely correlated with the occurrence of induced ONFH. Therefore, we speculated that caspase-3 may be employed as a predictor for fracture-induced ONFH. As biopsy could be achieved intraoperatively or could be CT-guided 
postoperatively, and the immunohistochemical staining of caspase- 3 is a relatively simple procedure, we believe that monitoring of the caspase- 3 expression in the femoral head is practical for clinical application. However, there were limitations to our study. More methods shoule be employed to detect apoptosis in addition to morphological observation and caspase-3 staining, such as the terminal deoxynucleotidyl transferase-mediated dUTP nick end-labeling (TUNEL) assay. An in vitro experiment is required to reflect the intrinsic mechanisms of fracture-induced apoptosis. Moreover, in order for caspase-3 to be used in clinical practice, more studies are required to observe the differences of biological and biomechanical properties between humans and the canine model.

\section{Acknowledgements}

The current research was financially supported by a Creative Research Scholarship for Ph.D. Candidates of Shanghai Jiao Tong University School of Medicine (No. BXJ2011038).

\section{References}

1. Bachiller F, Caballer AP and Portal LF: Avascular necrosis of the femoral head after femoral neck fracture. Clin Orthop Relat Res 399: 87-109, 2002.

2. Davidovitch RI, Jordan CJ, Egol KA and Vrahas MS: Challenges in the treatment of femoral neck fractures in the nonelderly adult J Trauma 68: 236-242, 2010.

3. Ly TV and Swiontkowski MF: Management of femoral neck fractures in young adults. Indian J Orthop 42: 3-12, 2008.

4. Nikolopoulos KE,Papadakis SA, Kateros KT,Themistocleous GS, Vlamis JA, Papagelopoulos PJ and Nikiforidis PA: Long-term outcome of patients with avascular necrosis, after internal fixation of femoral neck fractures. Injury 34: 525-528, 2003.

5. Haidukewych GJ: Salvage of failed treatment of femoral neck fractures. Instr Course Lect 58: 83-90, 2009.

6. Beris AE, Payatakes AH, Kostopoulos VK, et al: Non-union of femoral neck fractures with osteonecrosis of the femoral head: treatment with combined free vascularized fibular grafting and subtrochanteric valgus osteotomy. Orthop Clin North Am 35: 335-243, 2004.

7. Assouline-Dayan Y, Chang C, Greenspan A, Shoenfeld Y and Gershwin ME: Pathogenesis and natural history of osteonecrosis. Seminars Arthritis Rheum 32: 94-124, 2002.

8. Lausten GS, Hesse B, Thygesen V and Fogh J: Prediction of late complications of femoral neck fractures by scintigraph. Int Orthop 16: 260-264, 1992.

9. Watanabe Y, Terashima Y, Takenaka N, Kobayashi M and Matsushita T: Prediction of avascular necrosis of the femora head by measuring intramedullary oxygen tension after femoral neck fracture. J Orthop Trauma 21: 456-461, 2007.
10. Cho MR, Lee SW, Shin DK, Kim SK, Kim SY, Ko SB and Kwun KW: A predictive method for subsequent avascular necrosis of the femoral head (AVNFH) by observation of bleeding from the cannulated screw used for fixation of intracapsular femoral neck fractures. J Orthop Trauma 21: 158-164, 2007.

11. Sen RK, Tripathy SK, Gill SS, Verma N, Singh P and Radotra BD: Prediction of posttraumatic femoral head osteonecrosis by quantitative intraosseous aspirate and core biopsy analysis: a prospective study. Acta Orthop Belg 76: 486-492, 2010.

12. Ehlinger M, Moser T, Adam P, Bierry G, Gangi A, de Mathelin M and Bonnomet F: Early prediction of femoral head avascular necrosis following neck fracture. Orthop Traumatol Surg Res 97: 79-88, 2011.

13. Youm YS, Lee SY and Lee SH: Apoptosis in the osteonecrosis of the femoral head. Clin Orthop Surg 2: 250-255, 2010.

14. Calder JD, Buttery L, Revell PA, Pearse M and Polak JM: Apoptosis - a significant cause of bone cell death in osteonecrosis of the femoral head. J Bone Joint Surg Br 86: 1209-1213, 2004.

15. Zheng TS, Schlosser SF, Dao T, Hingorani R, Crispe IN, Boyer JL and Flavell RA: Caspase-3 controls both cytoplasmic and nuclear events associated with Fas-mediated apoptosis in vivo. Proc Natl Acad Sci USA 95: 13618-13623, 1998.

16. Petrigliano FA and Lieberman JR: Osteonecrosis of the hip: novel approach to evaluation and treatment. Clin Ortho Relat Res 465: 53-62, 2007.

17. Bhandari M, Tornetta P, Hanson B and Swiontkowski MF: Optimal internal fixation for femoral neck fractures: multiple screws or sliding hip screws? J Orthop Trauma 23: 403-407, 2009.

18. Duckworth AD, Bennet SJ, Aderinto J and Keating JF: Fixation of intracapsular fractures of the femoral neck in young patients: risk factors for failure. J Bone Joint Surg Br 93: 811-816, 2011.

19. Song KS: Displaced fracture of the femoral neck in children: open versus closed reduction. J Bone Joint Surg Br 92: 1148-1151, 2010.

20. Kakar S, Tornetta P III, Schemitsch EH, et al: Technical considerations in the operative management of femoral neck fractures in elderly patients: a multinational survey. J Trauma 63: 641-646, 2007.

21. Sato M, Sugano N, Ohzono K, Nomura S, Kitamura Y, Tsukamoto Y and Ogawa S: Apoptosis and expression of stress protein (ORP150, HO1) during development of ischemic osteonecrosis in the rat. J Bone Joint Surg Br 83: 751-759, 2001.

22. Weinstein RS, Nicholas RW and Manolagas SC: Apoptosis of osteocytes in glucocorticoid-induced osteonecrosis of the hip. J Clin Endocrinol Metab 85: 2907-2912, 2000.

23. Kikuyama A, Fukuda K, Mori S, Okada M, Yamaguchi H and Hamanishi C: Hydrogen peroxide induces apoptosis of osteocytes: involvement of calcium ion and caspase activity. Calcif Tissue Int 71: 243-248, 2002.

24. Zalavras C, Shah S, Birnbaum MJ and Frenkel B: Role of apoptosis in glucocorticoid-induced osteoporosis and osteonecrosis. Crit Rev Eukaryot Gene Expr 13: 221-235, 2003.

25. Kabata T, Kubo T, Matsumoto T, Nishino M, Tomita K, Katsuda S, Horii T, Uto N and Kitajima I: Apoptotic cell death in steroid induced osteonecrosis: an experimental study in rabbits. J Rheumatol 27: 2166-2171, 2002. 